\title{
Design e Antropometria: diferenciação estratégica
}

\author{
Design and Antropometrics: Strategic Diferentiation
}
SCHOENARDIE, Rodrigo Petry; Mestrando em Gestão Estratégica de Design; Universidade Federal de Santa Catarina - UFSC rodrigops@gmail.com

TEIXEIRA, Clarissa Stefani, Doutoranda em Engenharia de Produção; Universidade Federal de Santa Catarina - UFSC clastefani@gmail.com

MERINO, Eugenio Andrés Díaz; Doutor em Engenharia; Docente da Universidade Federal de Santa Catarina - UFSC merino@deps.ufsc.br

\begin{abstract}
Resumo
O presente trabalho busca demonstrar, por meio de uma pesquisa exploratória e de revisão bibliográfica, a importância estratégica dos parâmetros antropométricos para o design de produto. Evidencia também os diversos dados antropométricos disponíveis e sua validade para o desenvolvimento de produtos enfocando a diferenciação. Conclui-se que a incorporação da antropometria no desenvolvimento de produtos permite um diferencial mercadológico, assumindo valor estratégico dentro do processo de design.
\end{abstract}

Palavras-chave: Design; Gestão de Design; Antropometria; Produtos; Diferenciação.

\begin{abstract}
This paper, through an exploratory research and literature review seeks to demonstrate product design anthropometric parameters strategic importance. The paper seeks also to reveal the various anthropometric data available and its product development validity focusing on differentiation. It concludes that anthropometry incorporation in product development allows market differential, assuming strategic value within the design process.
\end{abstract}

Keywords: Design; Design Management; Anthropometry; Products; Differentiation.

\section{Considerações Iniciais}

Etimologicamente, a ergonomia tem sua origem nas palavras gregas ERGON (trabalho) e NOMOS (regras). Pode-se assim ser considerada como o estudo das leis do trabalho. Nos Estados Unidos, a Ergonomia também consta como sinônimo o termo human factor (fatores humanos) (DULL; WEERDMEESTER, 2004). Em seu surgimento, envolve a abordagem do trabalho humano e suas interações no contexto social e tecnológico e demonstração da complexidade da situação de trabalho e os múltiplos fatores envolvidos nas práticas profissionais (ABRAHÃO; PINHO, 2002). Portanto, as contribuições da Ergonomia enquanto área de interesse para melhorias das condições humanas em geral, abrange não apenas o trabalho executado a partir 
Rodrigo P. Schoenardie; Clarissa S.Teixeira; Eugenio A. D. Merino

de máquinas e equipamentos, mas também nas demais situações do "fazer" humano, onde ocorre o relacionamento entre o homem e uma atividade produtiva (IIDA, 2005).

De maneira geral e a partir das indicações das associações que abordam a Ergonomia $\left(\mathrm{ABERGO}^{1}\right.$ e $\mathrm{IEA}^{2}$ ) podem ser identificados três domínios de especialização da desta área: 1) organizacional, 2) cognitiva e 3) física. Estas especializações abordam as características específicas para cada sistema, assim como ilustra a Figura 1.
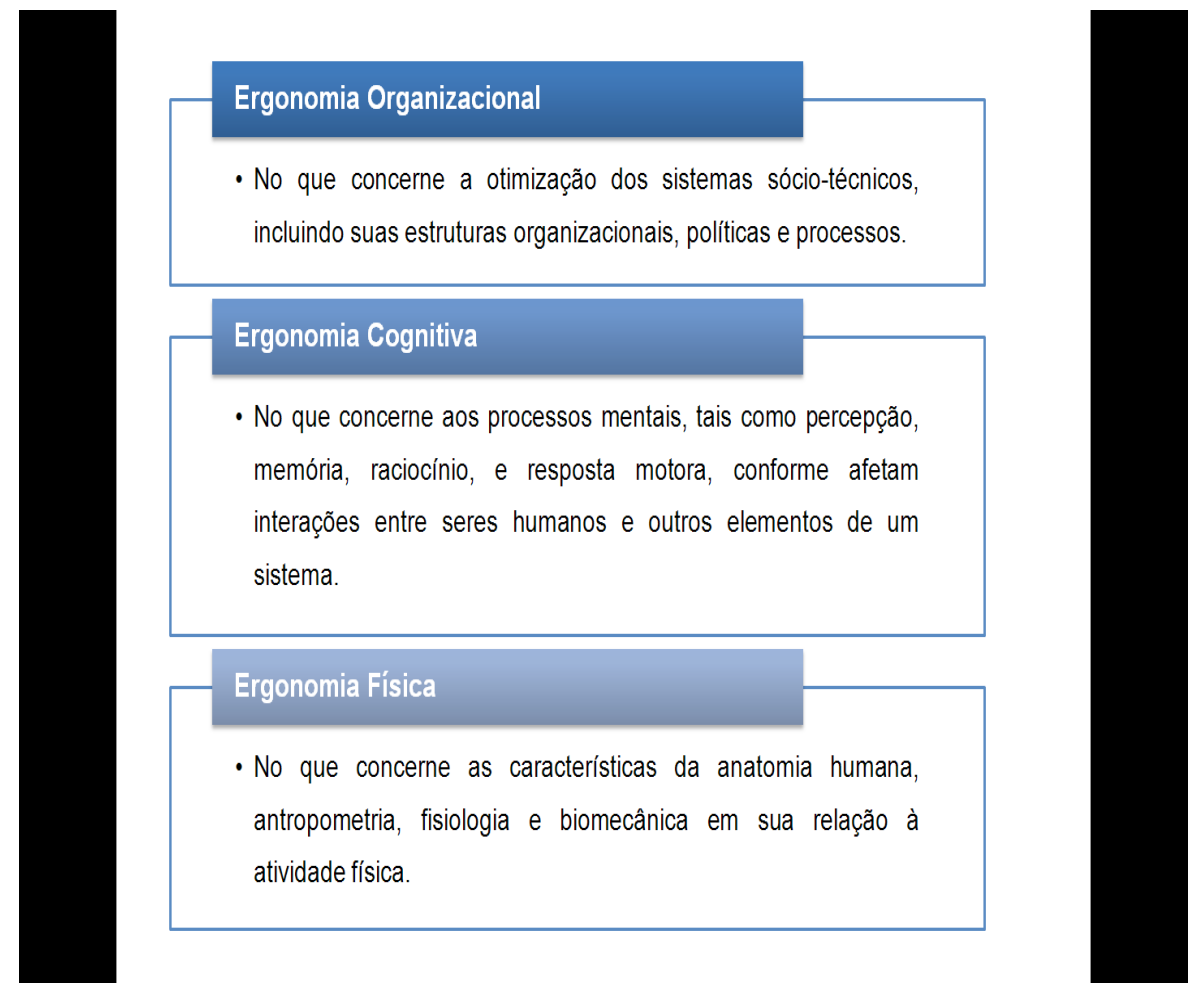

Figura 1 - Domínios especializados da ergonomia, segundo a Classificação Internacional de Ergonomia.

Fonte: Merino e Teixeira (2010).

Dentre estes domínios, este trabalho enfocará a Ergonomia Física. Segundo o IEA (2010) a Ergonomia Física "concerne às características da anatomia humana, antropometria, fisiologia e biomecânica em sua relação à atividade física". Neste contexto, considerando a Ergonomia Física, uma das áreas que mais contribui estratégicamente com o design é a antropometria. Teixeira (2004) coloca que a antropometria estabelece parâmetros para os modelos corporais. Desta maneira, a Ergonomia apresenta contribuições fundamentais no que tange tanto os ajustes dos postos de trabalho e equipamentos, quanto à projeção de produtos adequados ao homem, ambas áreas de atuação do design.

De forma geral, as contribuições da Ergonomia são relevantes para as práticas projetuais. A área com o enfoque no design apresenta interesse para melhorias das condições humanas que abrange não apenas o trabalho executado a partir de máquinas e equipamentos, mas também as demais situações onde ocorre o relacionamento entre o homem e uma atividade produtiva (IIDA, 2005).

A melhoria dos aspectos funcionais, ergonômicos e visuais dos produtos visando o atendimento das necessidades do consumidor/usuário fazem parte das funções presentes no design (CONFEDERAÇÃO NACIONAL DAS INDÚSTRIAS, 1998). Além disso, o design apresenta importância frente à diferenciação dos produtos resultantes de seu processo (MOZOTA, 2011). Baseando-se nestas premissas, o presente estudo busca demonstrar, por meio de uma 
Design e Antropometria: diferenciação estratégica

revisão bibliográfica, a importância de parâmetros antropométricos para o design de produto identificando aspectos que podem ser considerados fragilidades, dentro do processo de desenvolvimento de produtos. Também busca evidenciar os diversos dados antropométricos disponíveis e sua validade para utilização no desenvolvimento de produtos sob enfoque da diferenciação.

\section{Metodologia}

Como metodologia, tem-se por base a taxonomia proposta por Gil (2002) e Vergara (2004), separando a classificação das pesquisas em dois grupos: quanto aos objetivos e quanto aos procedimentos técnicos utilizados. Assim, o presente trabalho é de caráter exploratório e bibliográfico. Como início, foi realizada uma pesquisa exploratória para definição dos objetivos e em seguida, como procedimento técnico, uma pesquisa bibliográfica foi realizada. O caráter exploratório do trabalho justifica-se, pois, por meio desta abordagem, o pesquisador é provido de conhecimentos diversos a respeito do assunto de estudo, tendo como "objetivo proporcionar maior familiaridade com o problema, com vistas a torná-lo mais explícito ou construir hipóteses" (GIL, 2002, p.41).

\section{Design e Gestão: Considerações para as bases antropométricas}

O design, dentro das diversas definições acerca do termo, tem evidenciado um duplo aspecto de atuação. Do ponto de vista operacional, o design limita-se ao projeto, de atuação pontual e sob demanda. Sob o enfoque estratégico, o design se incorpora no modelo de negócios e passa a ser implementado em todos os níveis de uma organização (GILLESPIE, 2002). Vale ressaltar que estes enfoques são complementares, pois sem oper acional, não há objeto para gestão e com a gestão, o operacional pode ser melhor efetivado.

A inserção do design dentro da estratégia da empresa é apontada por Martins e Merino (2008, p.229), que colocam que "o design caminha para uma nova configuração na qual projetos de forma isoladas e apenas operacionais vêm perdendo espaço para projetos sistêmicos e estratégicos". Para que isto ocorra, o processo do design deve ser gerido. Assim, pode-se expor que "a gestão de design tem a função de gerenciar recursos humanos e materiais, acompanhando o processo desde o surgimento de uma idéia até o seu lançamento no mercado" (CENTRO PORTUGUÊS DE DESIGN, 1997, p.14).

Dentro desta visão, a gestão de design se insere dentro de um contexto maior (SILVA, 2009), que passa pelas mudanças da gestão, que evoluiu de um modelo taylorista para um modelo de organização inteligente e flexível, que encoraja a tomada de riscos, a autonomia e a iniciativa. Este novo modelo, segundo Mozota (2011), apóia-se na gestão orientada ao cliente, no gerenciamento por projeto e na qualidade total. Pode-se complementar tal idéia, dizendo que a gestão de design é o "conjunto de técnicas de gestão empresarial dirigida a maximizar ao menor custo possível, a competitividade que obtém a empresa pela incorporação e utilização do design como instrumento de sua estratégia empresarial" (GIMENO, 2000, p.25).

Para o DMI $^{3}$ (DESIGN MANAGEMENT INSTITUTE, 2010), a gestão de design, de uma maneira objetiva, é o lado do negócio do design. A gestão de design abrange os processos em curso, as decisões de negócios e estratégias que permitem a inovação, a criação de produtos 
Rodrigo P. Schoenardie; Clarissa S.Teixeira; Eugenio A. D. Merino

efetivamente concebidos, serviços, comunicações, ambientes e marcas que melhoram a qualidade de vida do indivíduo. Além disso, pode-se dizer que a gestão de design vem a proporcionar o sucesso organizacional. Ainda segundo o Design Management Institute (2010), a gestão de design, em um nível mais profundo, visa conectar design, inovação, tecnologia, gestão e clientes para oferecer vantagens competitivas por meio do tripé da sustentabilidade, apresentado na Figura 2. Seria então a arte e a ciência de capacitação do design para melhorar a colaboração e a sinergia entre "design" e "negócios" para melhorar a eficácia do design.

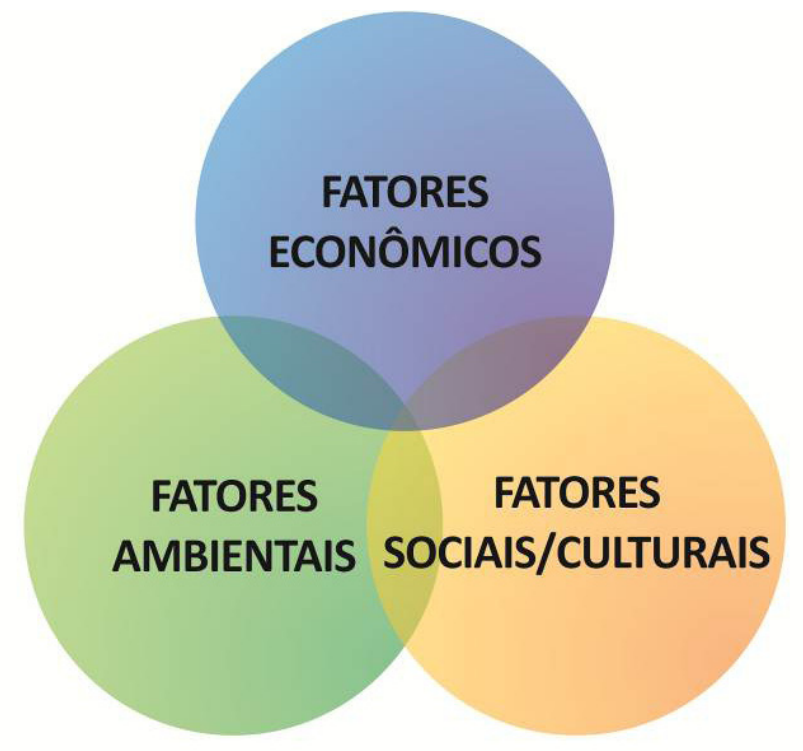

Figura 2- Tripé da Sustentabilidade.

Fonte: Adaptado de Design Management Institute (2010).

Segundo Mozota (2011), o conceito de Gestão de Design, surge nos anos 1960, na Inglaterra. Em 1966, Michael Ferrer apresentou uma nova função, a de gestor de design. Tal profissional deveria conduzir eficazmente os projetos e estabelecer uma boa relação entre a agência e seus clientes. Em 1975, Bill Hannon e o Massachusetts College of Art fundam, nos Estados Unidos, o Design Management Institute (DMI), cuja visão era inspirar o mundo a aprimorar a gestão dos processos de design nas organizações. Segundo o mesmo autor, a missão do Design Management Institute é ser uma autoridade internacional e a referência em matéria de gestão de design.

Para Bonsiepe (1997) somente nos anos 1990 a gestão de design torna-se uma temática central no discurso projetual do design, juntamente com a questão da sustentabilidade, entendendo-se assim o enfoque do DMI no tripé da sustentabilidade, como já mencionado.

Dentro da atuação da gestão de design, Best $(2006$, p.28) coloca que "o propósito da gestão de design é identificar e comunicar os caminhos pelos quais o design pode contribuir para agregar valor estratégico para a empresa". A mesma autora ainda define o papel do profissional que exercerá a função de gestor de design, sendo que "o gestor de design é responsável tanto pela coerência e consistência da mensagem de design da organização, quanto por assegurar que ela esteja alinhada à sua estratégia de negócios".

O uso de informações mostra-se estrategicamente importante dentro da gestão de design. Neste aspecto, um dos objetivos recorrentes em projetos de design é a busca por diferenciação. Selecionar e gerir as informações pertinentes e estratégicas dentro do processo de projeto pode contribuir para tal diferenciação. Para Mozota (2011) o design posiciona-se dentro de quatro poderes num modelo de gestão: como diferenciador, integrador, transformador

Projética Revista Científica de Design I Universidade Estadual de Londrina I V.2 I N.2 I Dezembro 2011 
Design e Antropometria: diferenciação estratégica

e como bom negócio. A autora coloca a vantagem competitiva assumindo duas formas: design como diferenciador e design como coordenador ou integrador. Magalhães (1997) indica que design como estratégia competitiva, precisa da integração das informações interdisciplinares do processo de desenvolvimento de produto.

De acordo com Mozota (2011) a diferenciação de um produto ocorre de acordo com oito dimensões: função, desempenho, conformidade, durabilidade, confiabilidade, capacidade de ser recuperado, estilo e serviço. A partir desta definição, pode-se apontar que os dados antropométricos são de primária importância para ao menos, mas não somente, quatro destes ítens, sendo eles: função, desempenho, conformidade e confiabilidade.

Um produto exerce plenamente sua função se for, dentre outros requisitos, bem dimensionado, tanto técnica quanto antropometricamente. Atrelado a cumprir a função está o desempenho, que pode ser otimizado a partir da consideração de dados antropométricos corretos. Sua conformidade além de atender normatizações técnicas e de segurança, deveria contemplar a antropometria. E, finalmente, pode-se citar a confiabilidade ao executar sua função que deriva da percepção dos ítens anteriores pelo usuário.

Assim, pode-se considerar que a antropometria tem valor fundamental para o design. Porém, as informações antropométricas disponíveis nem sempre são pertinentes a todos os projetos e nem sempre estão em consonância com os mesmos. Selecionar e saber gerir esta diversidade de dados se constitui em um desafio dentro do processo de design.

No que concerne à medidas antropométricas específicas, esta variedade de dados deve ser filtrada e estar alinhada com as definições presentes no briefing ${ }^{4}$ do projeto. Um briefing de design de qualidade precisa conter informações específicas e estratégicas (PHILIPS, 2008). Além disso, observa-se que a identificação de quais dados antropométricos devem ser considerados para a prática de projeto também depende de tais informações.

A diversidade de dados e suas populações consideradas podem levar ao uso de informações equivocadas. Sell (2002) indica que a população brasileira por suas características, que englobam misturas de diversas raças e com condições sociais distintas entre as grandes regiões do país, dificulta o projeto dos postos de trabalho no qual todos os trabalhadores devem estar bem acomodados e dificulta a produção dos produtos. Ainda neste sentido, IIDA (2005) demonstra a diversidade antropométrica e as diferentes proporções corporais de indivíduos de diferentes etnias (americanos, japoneses e brasileiros), assim como ilustra a Figura 3.

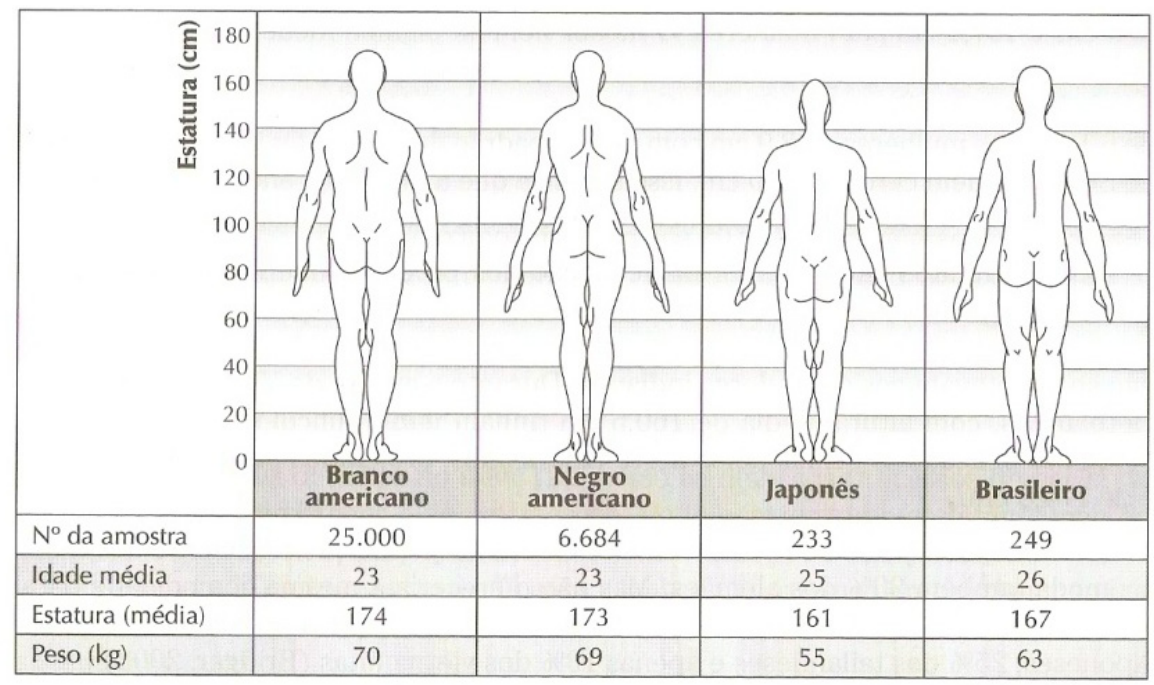

Figura 3- Diferenças entre as proporções corporais de indivíduos de diferentes etnias.

Fonte: IIDA (2005). 
Rodrigo P. Schoenardie; Clarissa S.Teixeira; Eugenio A. D. Merino

Mesmo focando em uma população específica, a aquisição de dados considerados corretos e pertinentes para o projeto ainda é um desafio. Especificando apenas a população brasileira, Felisberto (2001) aponta que as condições histórico-geográficas não favorecem o planejamento projetual quando as características antropométricas são consideradas. Segundo o autor é ainda muito difícil definir um padrão brasileiro, caracterizando as poucas referências disponibilizadas e encontradas na literatura (tanto nacional quanto internacional). Como agravantes poucos são os dados encontrados que podem ser considerados como representativos de indivíduos específicos, tornando sua utilização pouco confiável, o que traz prejuízos ao resultado e ao processo de design.

Ainda sobre esse aspecto, pode-se dizer que não foram encontrados estudos que identifiquem as características antropométricas de todo o corpo humano, considerando uma amostra significativa da população brasileira. De forma geral, os estudos remetem a indivíduos específicos (MINETTI, 2002; PEREIRA, 2006) e consideram poucas variáveis antropométricas, sendo encontradas principalmente a massa e a estatura corporal (GUIMARÃES, 2002; MARTÍNEZCARRIÓN, 2009). De fato, pode-se dizer que do ponto de vista projetual estes dados possuem importância, mas quando consideradas unicamente, demonstram ser um embasamento frágil para projetos.

A variação dos dados, devido à restrição de população considerada, também pode trazer prejuízos e dificuldades a um projeto. Menin e Paschoarelli (2006) encontrou em seu estudo diferenças nas medidas antropométricas considerando as normas e uma amostra de indivíduos obesos do Brasil. Além disso, o estudo de Guimarães (2002) apresenta as diferenças entre a estatura de trabalhadores do Rio Grande do Sul e os dados do Instituto Nacional de Tecnologia (INT) na ordem de 2,4\%, sendo o percentil 50 da estatura do INT equivalente ao percentil 31 do Rio Grande do Sul. Posteriormente, os autores descrevem que a maior diferença apareceu no percentil 95 e 99, considerando a massa corporal, onde houve aumento de aproximadamente $11,4 \%$ de diferenças, o que equivale a $10 \mathrm{~kg}$. Resumidamente, observa-se que estas diferenças se apresentam como importantes pontos de análise e considerações no planejamento de qualquer projeto visto as diferenças encontradas.

Adotar normas e padrões internacionais poderia parecer uma solução para o embasamento projetual. Porém, Felisberto (2001) aponta que tais dados apresentam os mesmos problemas da realidade brasileira. Hanson (2009), por exemplo, indica que mesmo considerando a população sueca, mais homogênea comparada à brasileira, a necessidades de atualizações para a adaptação de novos produtos é eminente, pois ao comparar dados mais atuais, do período de 2008 , com outros coletados na década de 60 , verificou-se variação de até $48,4 \%$ em determinados dados antropométricos. Outros fatores também devem influenciar na decisão dos dados antropométricos a serem considerados, como por exemplo, os efeitos da idade para as variáveis antropométricas, assim como indica Mokdad (2002).

De forma geral, pode-se dizer que todas as regiões corporais são importantes para a projeção de produtos. As medidas das mãos, por exemplo, influenciam todo e qualquer projeto que tenha necessidade de interação com esta região corporal. No entanto, as medidas dos polegares (falange distal do polegar), para qualquer população, não foi encontrada na literatura sendo uma fragilidade para o processo do design, assim como indica Schoenardie et al. (2010) que realizaram o redesign de uma chave de fenda, mostrado na Figura 4. Tal lacuna provavelmente não é a única no que se refere a dados específicos que podem auxiliar em projetos de produtos.

Projética Revista Científica de Design I Universidade Estadual de Londrina I V.2 I N.2 I Dezembro 2011 


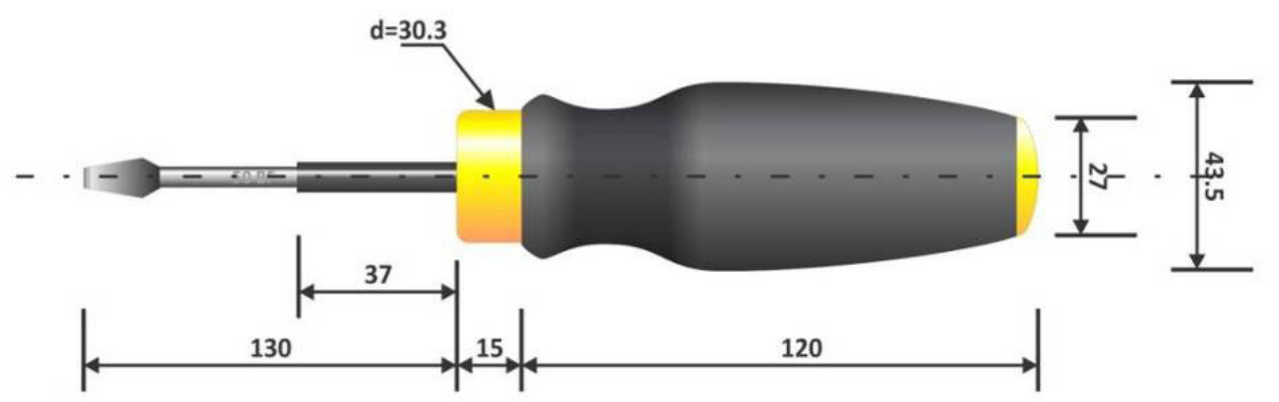

todas as medidas em $\mathrm{mm}$

Figura 4 - Modelo conceitual de chave de fenda

Fonte: Schoenardie et al. (2010).

A consideração das diferenças de dados e suas origens permitem iniciativas como as adotadas pelas indústrias de óculos. Ball et al. (2010) indicam que as diferenças antropométricas entre a população ocidental e oriental são relevantes, principalmente considerando as medidas da cabeça, tais como ponte nasal, distância entre os olhos e geometria geral da face. A Figura 5 exemplifica os dados coletados na pesquisa.

\section{Cabeça}

Chinesa
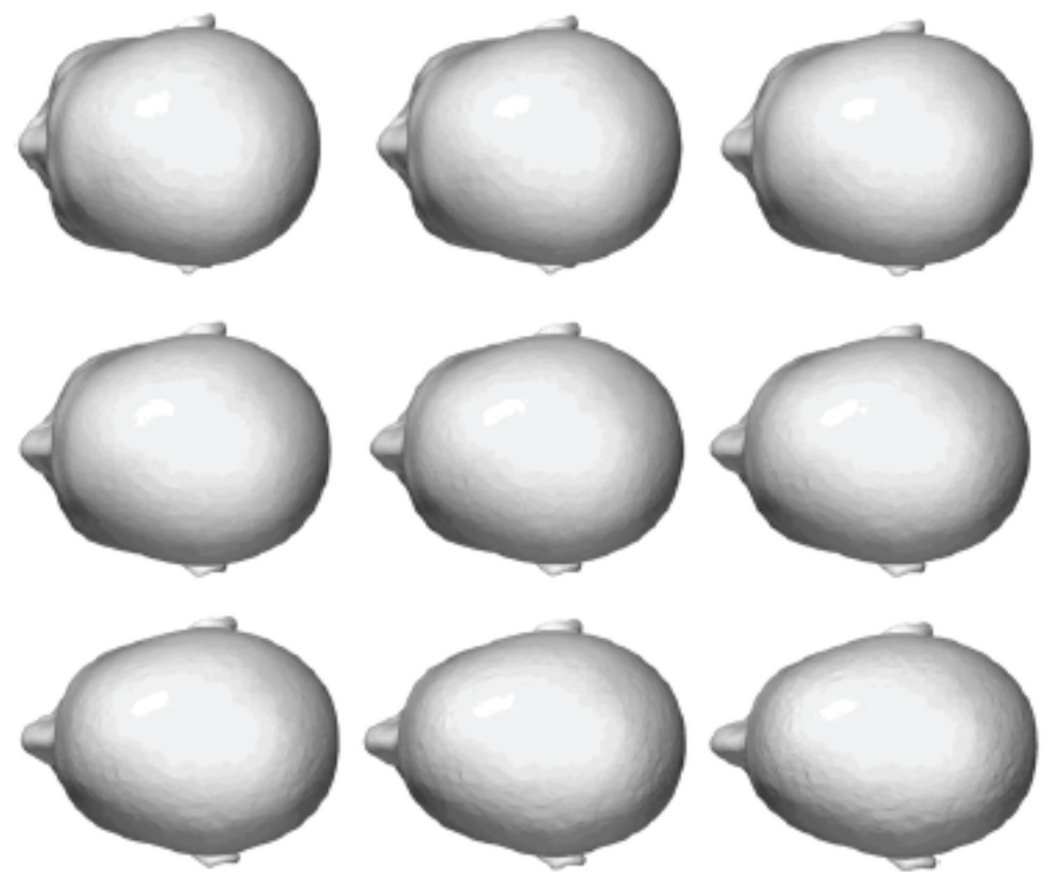

\section{Cabeça \\ Caucasiana}

Figura 5 - Diferença de forma e tamanho da cabeça de indivíduos chineses e caucasianos Fonte: Ball et al. (2010).

Os mesmos autores relatam a criação da denominação "Asian Fit" pela indústria de óculos, como diferencial gerado pelo design com relação direta a antropometria. Neste caso, os dados antropométricos evidenciando as diferenças entre as populações foram considerados no projeto de design, trazendo a diferenciação pela melhor adequação do produto ao usuário. 
Rodrigo P. Schoenardie; Clarissa S.Teixeira; Eugenio A. D. Merino

Nas figuras que seguem, fica explícita a aplicação de dados antropométricos específicos neste mesmo contexto, em produtos de mercado focados em consumidores de populações distintas, utilizando o "Asian Fit" como diferencial. Com demonstrado na Figura 6, os dados levaram a uma redução da medida entre os apoios dos óculos na ponte nasal. Dados desta mesma área resultaram na consideração de uma maior distância entre os apoios e a estruturados óculos, como mostrado na figura 7.

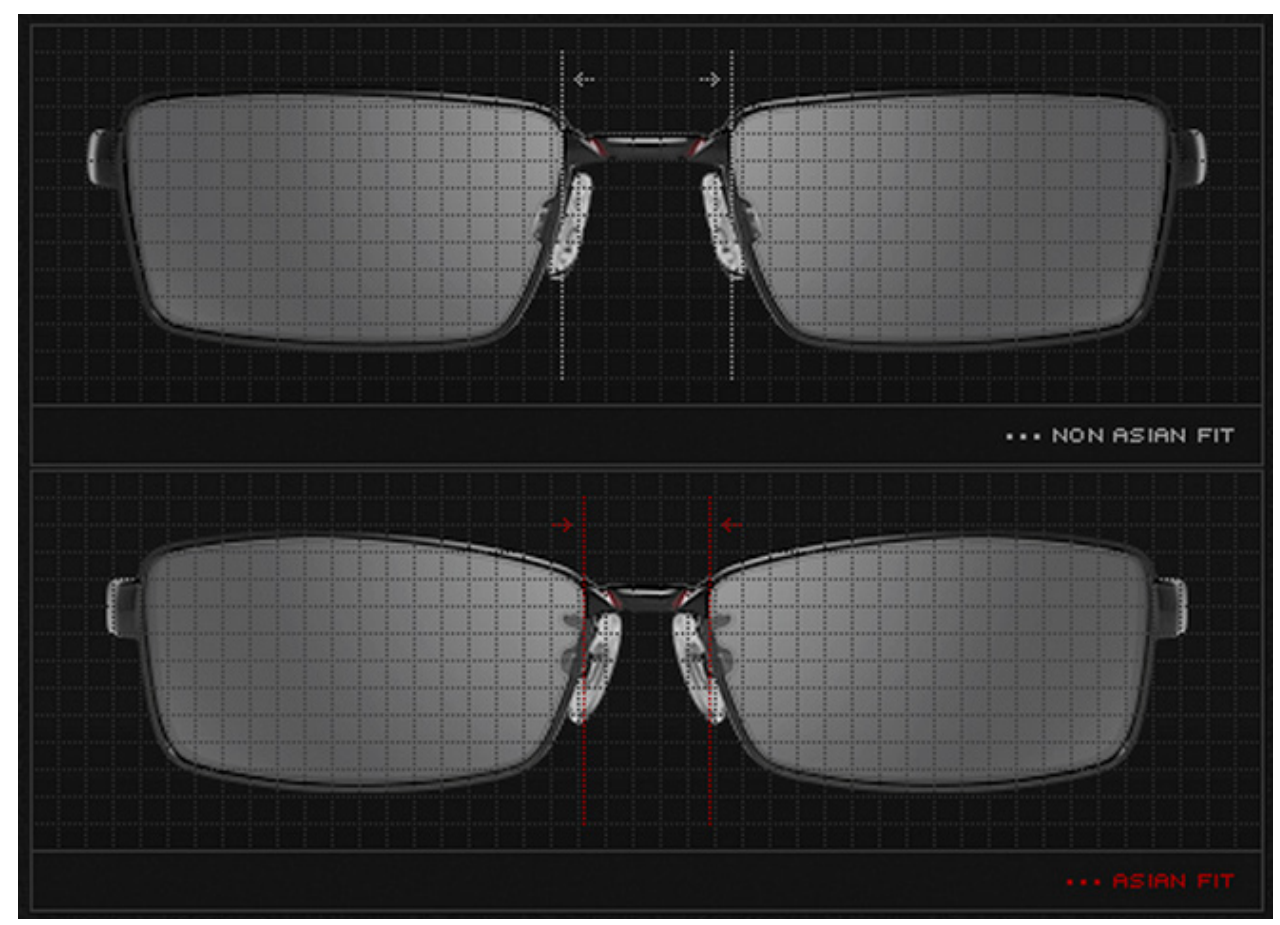

Figura 6- Modelo de óculos "Asian Fit" comparado com óculos tradicionais em relação à largura do posicionamento dos apoios na ponte nasal.

Fonte: Oakley (2010).

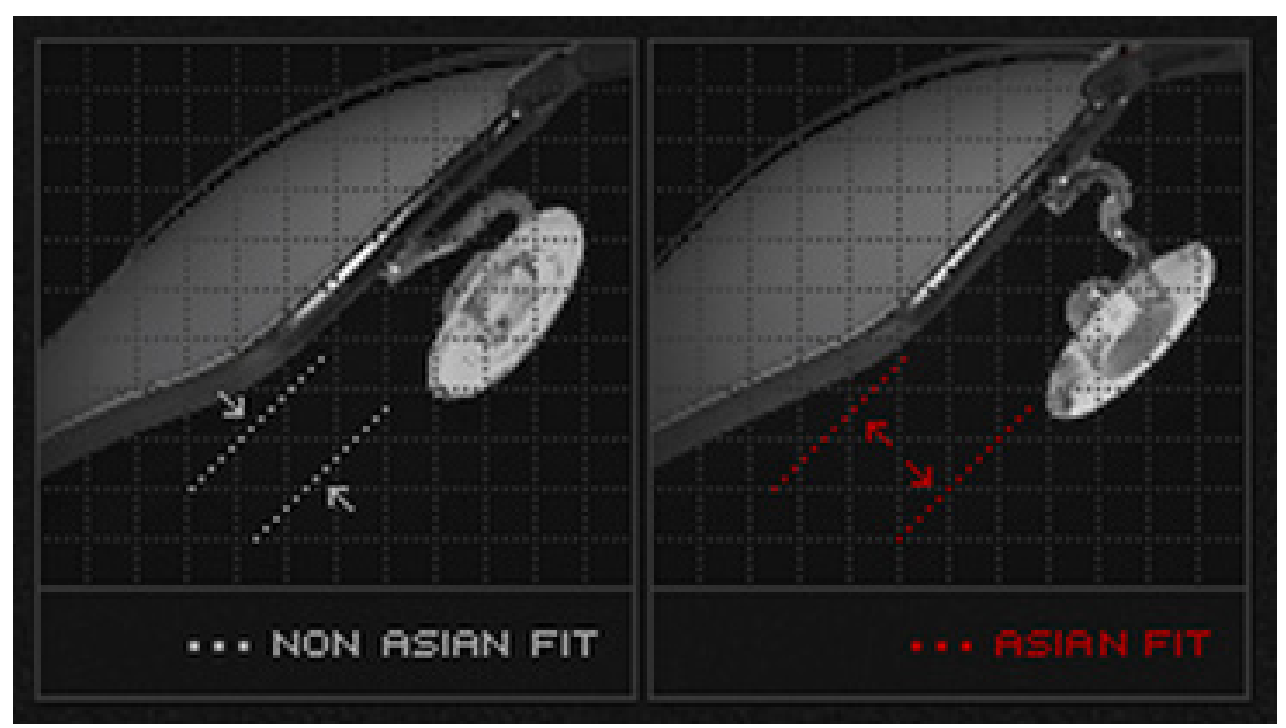

Figura 7- Modelo de óculos "Asian Fit" comparado com óculos tradicionais em relação à distância do posicionamento dos apoios na ponte nasal e a estrutura do óculos.

Fonte: Oakley (2010). 
Design e Antropometria: diferenciação estratégica

No caso específico dos óculos, outras variáveis antropométricas também influenciaram estrategicamente o design final dos produtos. A Figura 8 mostra a diferença na área de apoio na ponte nasal. A conformação diferenciada da geometria da face da população asiática levou também a possibilidade da aplicação dos dados em outros produtos, como óculos de proteção para a prática de esportes de inverno, como demonstrado na figura 9.

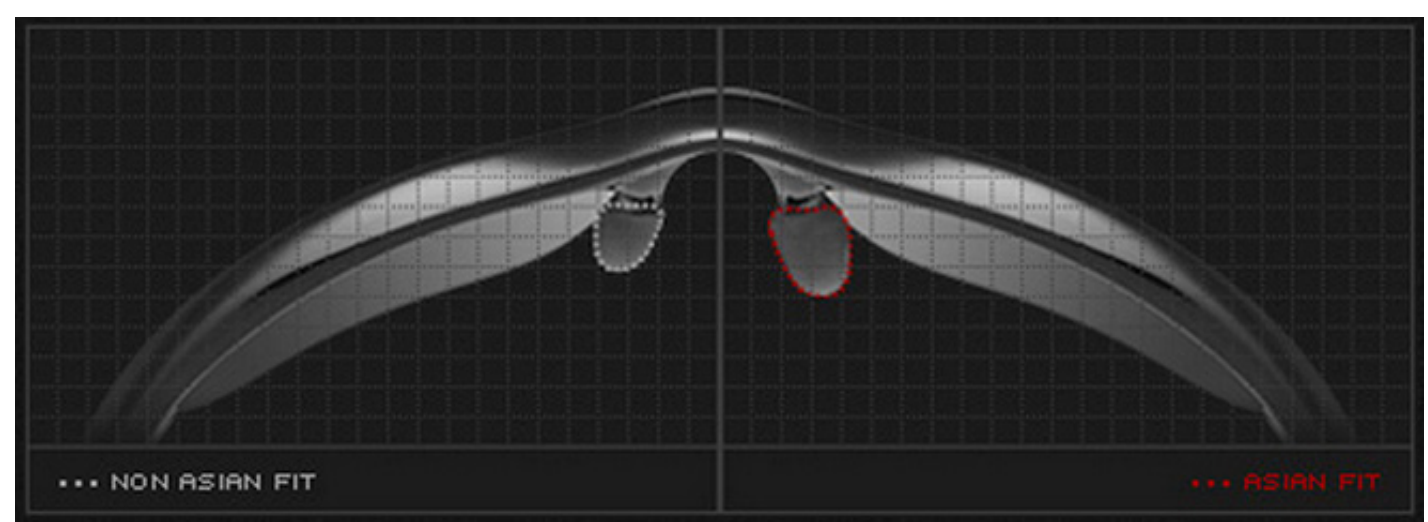

Figura 8- Modelo de óculos "Asian Fit" comparado com óculos tradicionais, com relação a área de contato do apoio na ponte nasal.

Fonte: Oakley (2010).

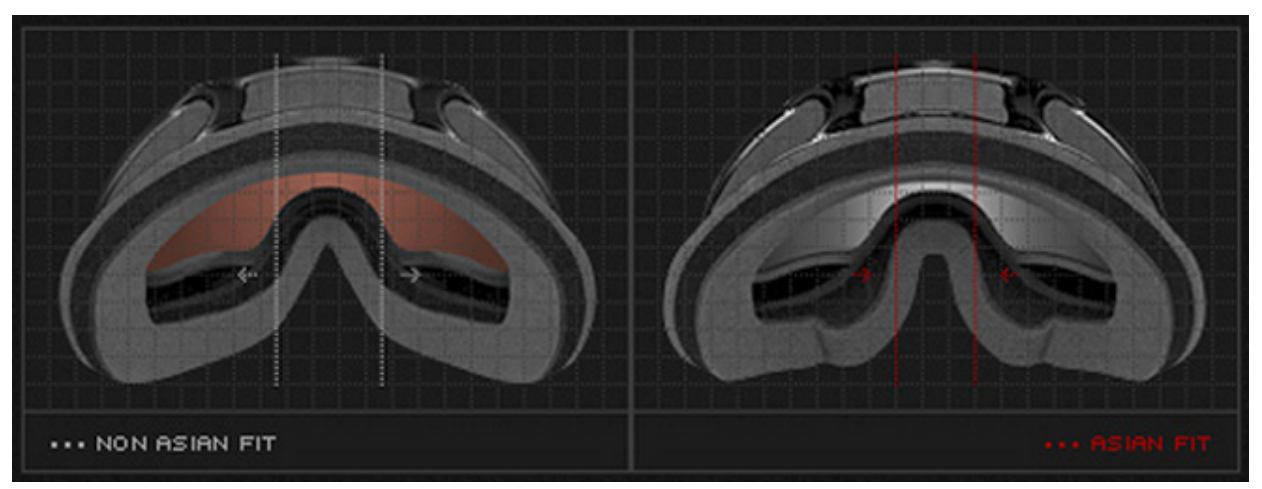

Figura 9- Modelo de óculos "Asian Fit" comparado com óculos tradicionais em relação a geometria da face.

Fonte: Oakley (2010).

Dentro deste mesmo contexto, o trabalho de Ramires et al (2011) traz um levantamento de tipologia e antropometria visual, com dados da população nacional, ressaltando as diferenças significativas entre os sexos e os percentis dentro de um mesmo sexo, considerando as medidas da face. Desta forma, evidencia-se que há diferenças consideráveis nestes dados para as práticas projetuais e estas precisam ser avaliadas para o processo de desenvolvimento do produto.

\section{Considerações Finais}

De forma geral, pode-se dizer que a gestão de design, dentre suas diversas atribuições e definições, preocupa-se com o fluxo e seleção das informações relevantes ao processo operacional do design. Tal processo pode buscar em sua formalização final a diferenciação dos produtos resultantes. Dentro deste aspecto, a antropometria é uma área fundamental 
Rodrigo P. Schoenardie; Clarissa S.Teixeira; Eugenio A. D. Merino

para o design de produtos. Ainda neste sentido, selecionar as informações corretas e pertinentes apresenta-se como estratégia importante dentro do processo da gestão de design. A necessidade de seleção e filtragem corretas, alinhadas ao briefing de projeto, evidencia a importância que o processo de gestão possui no design. A seleção dos dados antropométricos válidos e precisos é parte fundamental do processo e chave para o sucesso do produto.

Neste sentido, a coleta e atualização sistemática dos dados antropométricos também são importantes. Ademais, mesmo com pequenas variações nos dados antropométricos pode haver interferências nas atividades e assim causar prejuízos diretamente associados à saúde, a segurança, a percepção de conforto e a produtividade dos indivíduos. Assim, as medidas a serem consideradas para qualquer tipo de estudo ou aplicação devem estar associadas às necessidades do projeto considerando seus usuários. Também vale salientar que a coleta de dados em antropometria deve ser realizada com instrumentos adequados e pessoas treinadas para tal fim.

A incorporação eficaz e eficiente da antropometria, tanto no projeto de concepção quanto no de correção é fundamental para o sucesso do processo de design. Tal incorporação permite inclusive o diferencial mercadológico, como já evidenciado. A antropometria assume assim, valor estratégico dentro do processo de design, na busca da diferenciação e resolução de problemas. No entanto, a busca por dados mais próximos da realidade da população ainda é necessária.

\section{Notas}

1 Associação Brasileira de Ergonomia. Disponível em: http://www.abergo.org.br

2 International Ergonomics Association (Associação Internacional de Ergonomia). Disponível em:http://www. iea.cc

3 Design Management Institute (Instituto de Gestão de Design). Disponível em: http://www.dmi.org

4 Segundo Philips (2008) o briefing é a reunião de "todas as informações relevantes aos interessados no projeto."

\section{Referências}

ABRAHÃO, Júlia; PINHO, Diana. As transformações do trabalho e desafios teórico-metodológicos da ergonomia. Estudos de Psicologia, v. 7, p. 45-52, 2002.

BALL, Roger; SHU, Chang; XI, Pencheng; RIOUX, Marc; LUXIMON, Yan; MOLENBROEK, Johan. A comparison between Chinese and Caucasian head shapes. Applied Ergonomics, v. 41, p. 832839, 2010.

BEST, Kathrin. Design Management - Managing Design Strategy, Process and Implementation. Switzerland: AVA, 2006.

BONSIEPE, Gui. Design: do material ao digital. Florianópolis: FIESC/IEL, 1997.

CONFEDERAÇÃO NACIONAL DAS INDÚSTRIAS. A Importância do Design para sua Empresa. Brasília, 1998.

CENTRO PORTUGUÊS DE DESIGN (Portugal). Manual de Gestão de Design. Porto: Porto Ed.,

Projética Revista Científica de Design I Universidade Estadual de Londrina I V.2 I N.2 I Dezembro 2011 
Design e Antropometria: diferenciação estratégica

1997.

DESIGN MANAGEMENT INSTITUTE. What is Design Management? 2010. Disponível em:<http:// www.dmi.org/dmi/html/aboutdmi/design_management.htm>. Acesso em: 28 maio 2011.

DULL, Jan; WEERDMEESTER, Bernard. Ergonomia prática. São Paulo: Edgard Blücher, 2004.

FELISBERTO, Luiz Carlos; PASCHOARELLI, Luis Carlos. Dimensionamento preliminar de postos de trabalho e produtos - modelos antropométricos em escala. In: ENCONTRO NACIONAL DE ENGENHARIA DE PRODUÇÃO; Anais... INTERNATIONAL CONFERENCE ON INDUSTRIAL ENGINEERING E OPERATIONS MANAGEMENT, 7., 2001, Salvador. Proceedings, 2001. 1 CD ROM.

GIL, Antônio Carlos. Como elaborar projetos de pesquisa. 4. ed. São Paulo: Atlas, 2002.

GILLESPIE, Brian. Strategic Design Management and the Role of Consulting. 2002. Disponível em:<http://www.designingbusiness.com/BrianG_SDM_ResearchReport.pdf>. Acesso em: 10 mar. 2011.

GIMENO, José María Iváñez. La gestión del diseño en la empresa. Madrid: Mcgraw-hill de Management, 2000.

GUIMARÃES, Lia Buarque de Macedo; BIASOLLI, Patrícia Klaser. Levantamento antropométrico: o Brasil ainda precisa ter o seu? In: CONGRESSO INTERNACIONAL DE ERGONOMIA E USABILIDADE DE INTERFACES HUMANO-TECNOLOGIA: Produtos, Programas, Informação, Ambiente Construído Ergodesign, 2., 2002, Rio de Janeiro. Anais..., II Ergodesign. Rio de Janeiro: Departamento de Artes e Design PUC-Rio, 2002. 1CD ROM.

HANSON, Lars; SPERLING, Lena; GARD, Gunvor; IPSEN, Staffan; VERGARA, Cindy Olivares. Swedish anthropometrics for product and workplace design. Applied Ergonomics, v. 40, p. 797806, 2009.

IIDA, Itiro. Ergonomia projeto e produção. São Paulo: Edgar Blucher, 2005.

MAGALHÃES, Claudio Freitas de. Design Estratégico: Integração e Ação do Design Industrial dentro das Empresas. Rio de Janeiro: SENAI/ DN, 1997.

MARTíNEZ-CARRIÓN, José Miguel; PUCHE-GIL, Javier. La estatura de los españoles en el espejo francés: una historia antropométrica comparada. Documentos de Trabajo -AEHE, n. 0911, p.140, 2009.

MARTINS, Rosane Fonseca de Freitas; MERINO, Eugenio Andres Dias. A Gestão de Design como Estratégia Organizacional. Londrina: EDUEL, 2008.

MENIN, Mariana; PASCHOARELLI; Luiz Carlos. Antropometria de obesos: Parâmetros para o design de produtos destinados à acessibilidade desta população. In: CONGRESSO DE PESQUISA E DESENVOLVIMENTO EM DESIGN. 7., 2006, Curitiba. Anais... Curitiba: Associação de Ensino e Pesquisa de Nível Superior de Design do Brasil, 2006. 1 CD-Rom.

MERINO, Eugenio Andres Dias; TEIXEIRA, Clarissa Stefani. Ergonomia e qualidade de vida nos sistemas de produção. Engenharia de Produção: Tópicos e Aplicações. Belém, v.1, p. 78-100, 
2010.

MINETTI, Luciano J.; SOUZA, Amaury P.; ALVES, José U.; FIEDLER, Nilton C. Estudo antropométrico de operadores de motosserra. Revista Brasileira de Engenharia Agrícola e Ambiental, Campina Grande, v. 6, n. 1, p. 166-170, 2002.

MOKDAD, M. Anthropometric study of Algerian farmers. International Journal of Industrial Ergonomics, n. 29, p. 331-341, 2002.

MOZOTA, Brigitte. Borja de. Gestão de design: usando o design para construir um valor de marca e inovação corporativa. Porto Alegre: Bookman, 2011.

OAKLEY. Asian Fit. 2010. Disponível em:<http://www.oakley.com/asian-fit>. Acesso em: 28 jun. 2011

PEREIRA, Érico Felden; TEIXEIRA, Clarissa Stefani. Proposta de valores normativos para avaliação da aptidão física em militares da Aeronáutica. Revista Brasileira de Educação Física e Esporte, v. 20, n. 4, p. 249-256, 2006.

PHILIPS, Peter L. Briefing: a gestão do projeto de design. São Paulo: Blucher, 2008.

RAMIRES, Rossana Ribeiro; FERREIRA, Léslie Piccolotto; MARCHESAN, Irene Queiroz; CATTONI, Débora Martins; SILVA, Marta Assumpção de Andrada e. Medidas faciais antropométricas de adultos segundo tipo facial e sexo. Rev. CEFAC, São Paulo, v. 13, n. 2, p. 245-252, 2011.

SCHOENARDIE, Rodrigo Petry; TEIXEIRA; Clarissa Stefani; MERINO, Giselle Schmit; MERINO, Eugenio Andres Dias. Parâmetros de projetos para a chave de fenda: considerações acerca dos quesitos de usabilidade e ergonomia. In: SIMPÓSIO DE ENGENHARIA DE PRODUÇÃO, 17., 2010, Bauru. Anais... São Paulo: UNESP, 2010

SELL, Ingeborg. Projeto do trabalho humano: melhorando as condições de trabalho. Florianópolis: UFSC, 2002.

SILVA, Cláudio Henrique da. Balanced Scorecard como ferramenta para gestão estratégica de design. 2009. 206 f. Dissertação (Mestrado) - UFSC, Florianópolis, 2009.

TEIXEIRA, Clarissa Stefani. Biomecânica. 2004. Trabalho de Conclusão de Curso (Graduação em Educação Física) - Universidade Federal de Santa Maria, 2004

VERGARA, Sylvia C. Projetos e Relatórios de Pesquisa em Administração. 5. ed. São Paulo: Atlas, 2004. 